\title{
Organic
} Electronics

\section{Anisotropic optical properties of single crystalline PTCDA studied by spectroscopic ellipsometry}

\author{
M.I. Alonso ${ }^{\mathrm{a}, *}$, M. Garriga ${ }^{\mathrm{a}}$, N. Karl ${ }^{\mathrm{b}}$, J.O. Ossó ${ }^{\mathrm{c}, \mathrm{a}}$, F. Schreiber ${ }^{\mathrm{c}, \mathrm{d}}$ \\ a Institut de Ciència de Materials de Barcelona, CSIC, Campus de la UAB, 08193 Bellaterra, Spain \\ b 3.Physikalisches Institut, Universität Stuttgart, 70550 Stuttgart, Germany \\ ${ }^{c}$ Max-Planck-Institut für Metallforschung, Heisenbergstr. 1, 70569 Stuttgart, Germany \\ ${ }^{\mathrm{d}}$ Institut für Theoretische und Angewandte Physik, Universität Stuttgart, 70550 Stuttgart, Germany
}

Received 2 August 2001; accepted 29 October 2001

\begin{abstract}
We report anisotropic spectra of 3,4,9,10-perylene tetracarboxylic dianhydride (PTCDA) in the optical energy range $1.5-3.7 \mathrm{eV}$, measured at room temperature by spectroscopic ellipsometry. The results were obtained on single crystals of $\alpha$-PTCDA grown by temperature gradient sublimation. Spectra were measured at highly symmetric positions of the plane of incidence relative to the sample crystallographic axes and are presented as pseudo-values of refractive index $\langle n\rangle$ and extinction coefficient $\langle k\rangle$, which reflect a very large anisotropy of these optical functions. The spectroscopic properties of the excited states in the molecular plane (1 02$)$ are analyzed; in particular, we identify clear Davydov splittings proving the formation of coherent excitons in the crystal. Out-of-plane, we observe a relatively narrow absorption peak at $\sim 2.9 \mathrm{eV}$ that is ascribed to a charge-transfer transition. (c) 2002 Elsevier Science B.V. All rights reserved.
\end{abstract}

PACS: 78.20.Ci; 78.40.Me; 71.35.Aa; 07.60.Fs

Keywords: Optical anisotropy; Complex dielectric function; Ellipsometry; 3,4,9,10-perylene tetracarboxylic dianhydride (PTCDA)

\section{Introduction}

Despite the increasing interest in layered organic low molecular weight materials with extended conjugated $\pi$-electron systems for applications in electronic and photonic devices, the fundamental optical properties of these materials are frequently not well known. The interacting $\pi$-electrons are not only the basis of the optical absorption in the visible part of the spectrum, but also of the relevant electronic and optoelectronic properties

\footnotetext{
* Corresponding author. Fax: +34-935-805-729.

E-mail address: isabel.alonso@icmab.es (M.I. Alonso).
}

of these materials. The red compound 3,4,9,10perylene tetracarboxylic dianhydride (PTCDA) is considered archetypical: Firstly, the sheet and stack crystal structure of this $\pi$-electron-rich planar molecule is a basis of well-defined growth [1]. Secondly, its unique electronic structure resulting from strong intermolecular $\pi$-electron overlap is a model example [2]. Thus, knowledge of the detailed optical spectra of the bulk material is most desirable, not only as a reference for characterization of PTCDA-containing heterostructures, but also to answer very basic questions on the nature of the excited states and the anisotropy of the optical properties of this class of materials. 
At present, the optical properties of single crystalline PTCDA are largely unknown. The lack of experimental data is mainly due to the difficulties of applying traditional polarized absorption or reflectivity methods to highly absorbing tiny crystals. Even to merely obtain the energies of electronic transitions, the use of modulation spectroscopies such as electro-absorption has wellknown difficulties of interpretation, since such spectra are usually validated by confronting them with numerical derivatives of absorption measurements. These limitations are known to be avoidable by using spectroscopic ellipsometry [3]. This measurement technique is self-normalizing, that is, no absolute intensity measurements are required, therefore neither a small size of a sample nor macroscopic roughness represent a principal hurdle. The typically high precision and low noise of the obtained spectra allow numerical derivatives to be made in order to obtain relevant parameters of electronic transitions by fitting to theoretical line shapes. In this context, ellipsometry is complementary to modulation spectroscopies. In this work we present ellipsometric spectra measured on two different sample crystal faces at different orientations of the plane of incidence. A preliminary analysis had been reported before, cf. [4].

The aim of this work is to contribute to the understanding of the anisotropic optical properties of PTCDA, both from the point of view of macroscopic optical response and of the underlying exciton states.

\section{Experimental details}

The samples used in this study were single crystals of $\alpha$-PTCDA obtained by temperature step sublimation under vacuum or by temperature gradient sublimation under a weak nitrogen gas stream at $\sim 1$ mbar, cf. [5]. The dimensions of the measured surfaces ranged from about $0.15 \times 0.30$ to $0.5 \times 2 \mathrm{~mm}^{2}$.

The structure of the crystals was checked in detail by X-ray diffraction. By comparison of our data for various Bragg reflections including (lll 102$)$ and others at oblique angles similar to [6], with the data for the $\alpha$ and $\beta$ [7-9] phases, the structural phase of the crystals used was determined to be $\alpha$. The measured faces of the crystals were checked by $\mathrm{X}$-ray diffraction and indexed. The crystal structure of PTCDA is drawn in Fig. 1.

Optical measurements were performed at room temperature using a spectral ellipsometer with rotating polarizer. As light source we used a $75 \mathrm{~W}$ high-pressure Xe arc lamp. The detection setup consisted of a double prism/grating monochromator of $750 \mathrm{~mm}$ equivalent focal length plus a multialkali photomultiplier tube. This setup covers a spectral range from 1.4 to $5.2 \mathrm{eV}$ with a resolution of about $1 \mathrm{meV}$. Since our light spot was larger than the measured crystals, these were adequately mounted for collecting only the light reflected by the surface of interest, discarding the rest. Therefore, due to the small size of some samples it was not always possible to record spectra in the full range, because of too low signal. We present spectra in the most relevant range from 1.5 to $3.7 \mathrm{eV}$. The sample holder was equipped with a goniometer that allowed azimuthal sample rotations. An alignment telescope was used to align the plane of incidence relative to the crystallographic coordinate system at a precision $\pm 1^{\circ}$. The angle of incidence of the optical beam was $\varphi=65^{\circ}$ and the analyser azimuth $A=30^{\circ}$. The data were recorded as a function of energy in steps chosen from 2 to $20 \mathrm{meV}$, depending on the required level of detail.

In this paper we report on measurements taken on two different crystallographic faces, (00 1) and (102). The former was an as-grown crystal facet, the latter obtained by cleavage. The conoscopic optical interference pattern viewed normal to the (10 2) lattice plane, see Fig. 2, displayed two optical axes with the acute bisectrix parallel to the reciprocal lattice vector (102), i.e., normal to the cleavage plane. The plane of the optical axes, which is an optical symmetry plane, is perpendicular to [0 10$]$, in accordance with the monoclinic (glide) mirror plane conferring symmetry to the ellipsometric data as well. In contrast to what might have been concluded from literature [10], PTCDA is optically biaxial, as follows already from the monoclinic crystal structure and from the fact that the long axes of the two symmetryequivalent molecules in the unit cell make an angle 

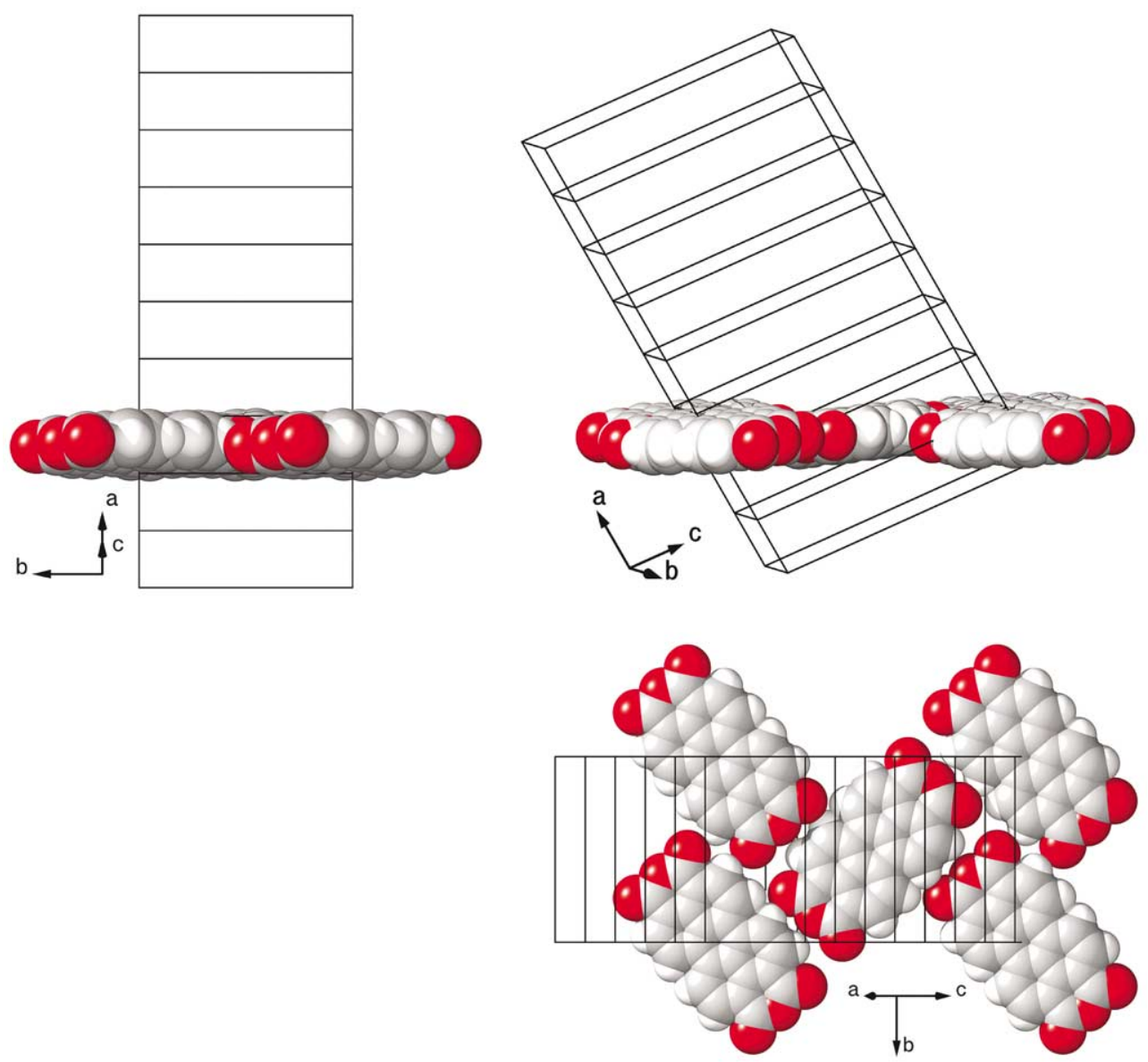

Fig. 1. 3,4,9,10-perylene tetracarboxylic dianhydride $\left(\mathrm{C}_{24} \mathrm{H}_{8} \mathrm{O}_{6}, \alpha\right.$-PTCDA), the monoclinic crystal structure, space group $P 2_{1} / c$ $(Z=2), a=0.372 \mathrm{~nm}, b=1.196 \mathrm{~nm}, c=1.734 \mathrm{~nm}, \beta_{c}=98.8^{\circ}$ [7]. Eight unit cells are shown to indicate the natural needle-like habit. Five molecules in the (1 $\left.\begin{array}{lll}1 & 2\end{array}\right)$ plane are drawn, the rest has been omitted for clarity. Upper left: projection along the glide mirror plane, which on the scale of the light wavelength is the mirror plane of the $2 / m$ point group symmetry of the crystal. Upper right: projection approximately down the two-fold rotation $b$-axis. Lower: projection perpendicular to the (10 2 10 plane.

on (1 02 ) distinctly different from (albeit close to) $90^{\circ}$.

It is well known that overlayers influence optical spectra [3]. In our case, no significant differences between spectra of either freshly cleaved or aged (lllll $\left.\begin{array}{ll}0 & 2\end{array}\right)$ surfaces with good specular appearance could be detected, indicating a high stability of this material under ambient conditions. However, longer time aged surfaces that displayed diffuse reflection exhibited ellipsometric spectra that indicated changes typical of contaminations or degradation.
For all studied crystal faces we chose the origin of the azimuthal angle of the crystal rotation about its normal $\beta=0$ when the plane of incidence contained the crystallographic $b$-axis (unique monoclinic axis). We acquired spectra at different settings $0 \leqslant \beta \leqslant 360^{\circ}$. In the (102) plane, only at the highly symmetric positions $\beta=0^{\circ}, 90^{\circ}, 180^{\circ}$ and $270^{\circ}$ we obtained spectra that were almost invariant with respect to the analyser azimuth [11]. This invariability is not strictly exact in the case of monoclinic symmetry [12] in contrast to the cases of higher symmetric crystal classes. 


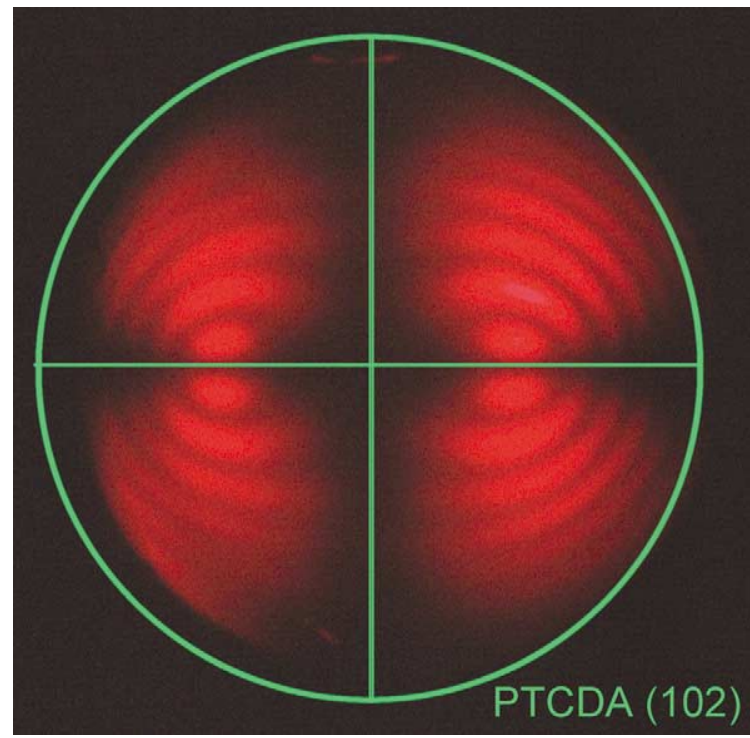

Fig. 2. Optical axes figure of $\alpha$-PTCDA, conoscopic view through a cleaved (102) platelet at $\lambda=750 \mathrm{~nm}$. The drawn lines correspond to the $\left[\begin{array}{lll}2 & 0 & 1\end{array}\right]$ direction (horizontal) and $\left[\begin{array}{lll}0 & 1 & 0\end{array}\right]$ direction (vertical).

\section{Optical function}

As the crystalline structure of $\alpha$-PTCDA is monoclinic, the optically biaxial crystal is characterized by three independent principal components of the dielectric tensor.

Denoting the three principal refractive indices as $n_{\alpha}, n_{\beta}$ and $n_{\gamma}$, it is concluded from the optical interferences, Fig. 2, and the thickness of the sample (ca. $35 \mu \mathrm{m}$ ) that - for $n_{\alpha}<n_{\beta}<n_{\gamma}-$ the $n_{\alpha}, n_{\beta}$ and $n_{\gamma}$ polarizations vibrate perpendicularly to the (1 $\left.\begin{array}{ll}1 & 2\end{array}\right)$ plane, and along [ $\left[\begin{array}{lll}0 & 1 & 0\end{array}\right]$ and $\left[\begin{array}{lll}2 & 0 & 1\end{array}\right]$ in the $\left(\begin{array}{lll}1 & 0 & 2\end{array}\right)$ plane, respectively; the $\left(\begin{array}{lll}1 & 0 & 2\end{array}\right)$ in-plane birefringence $\Delta n=n_{\gamma}-n_{\beta}$ amounts to ca. 0.03 at the wavelength of $750 \mathrm{~nm}$. This value compares well with the results of ellipsometric spectra (see below).

The exact treatment of the complex dielectric tensor in monoclinic symmetry is not well established yet. However, we can obtain approximate pseudo-values $\langle n\rangle$ and $\langle k\rangle$ of the optical functions by measuring in appropriate highly symmetric orientations [13]. In the spectral regions of low absorption the precision and accuracy of the experimental setup used (without a compensator) are known to be poor for determination of $\langle k\rangle$,
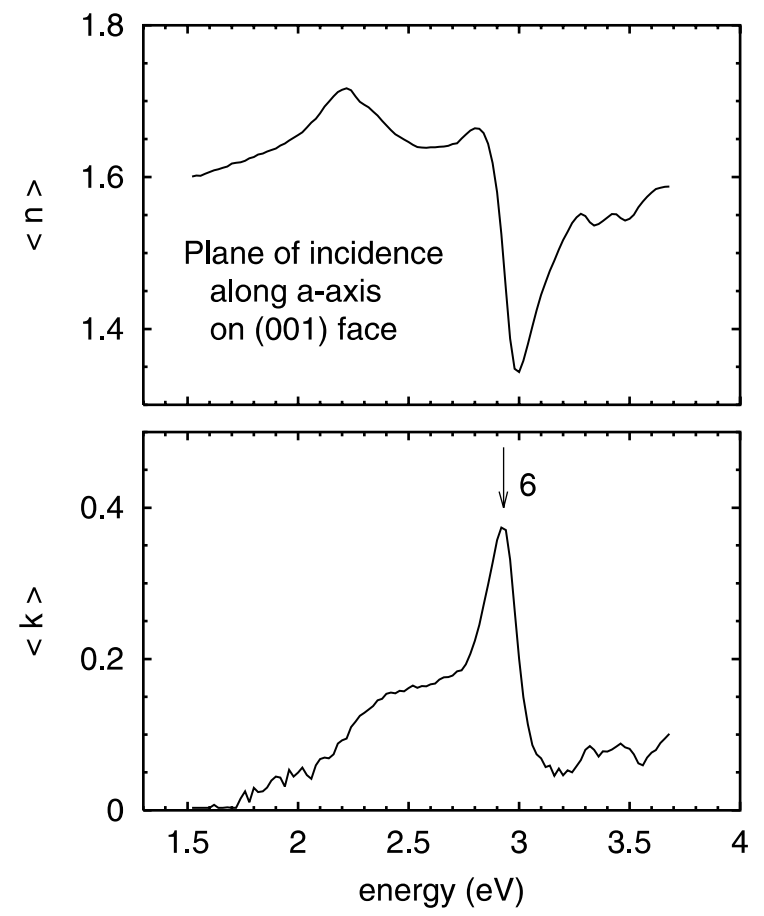

Fig. 3. Spectrum of the real $\langle n\rangle$ and imaginary part $\langle k\rangle$ of the refractive index obtained from a $\left(\begin{array}{ll}0 & 01\end{array}\right)$ face with the plane of incidence parallel to the $a$-axis. Therefore, these spectra reflect approximately the optical functions perpendicular to the molecular planes.

therefore $\langle k\rangle$ is noisy under these conditions (see Figs. 3-5). It should be emphasized, however, that in these regions $\langle n\rangle$ is essentially unaffected by this problem. In Fig. 3 we show the spectrum, displayed as $\langle n\rangle$ and $\langle k\rangle$, measured when the intersecting line between surface and plane of incidence is the $a$-axis. Thus, the dominant contribution to these optical functions comes from the projection of the dielectric tensor onto this intersecting line [13]. The molecules stack along this axis and, accordingly, the polarizability and hence the associated values of $\langle n\rangle \sim\left\langle n_{\alpha}\right\rangle$ are rather low. In contrast, the optical functions displayed in Figs. 4 and 5 arise mainly from the tensor projections onto the $b$-axis and its perpendicular [ $[201]$, respectively, on the common plane of the molecules. The largest anisotropy of the refractive index that we observe is of course this difference (in-plane versus out-of-plane) which in the visible transparency range is as large as $\Delta n \approx 2.60-1.60=1$. 

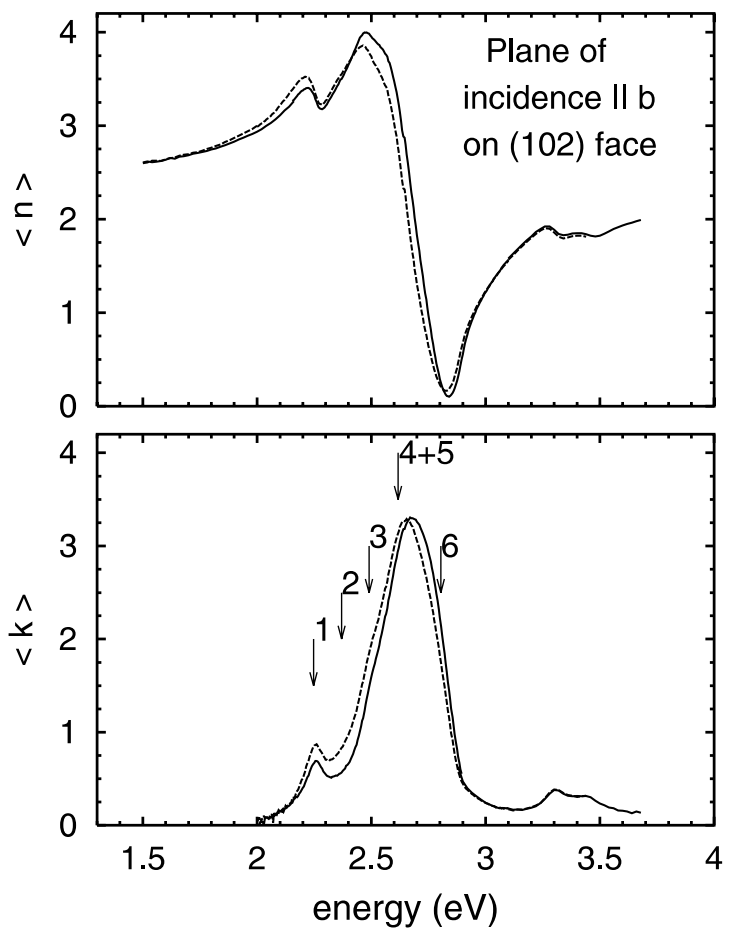

Fig. 4. Spectra from a $\left(\begin{array}{ll}1 & 02\end{array}\right)$ face with the plane of incidence parallel to the $b$-axis. The measurements from sample azimuths $\beta=0$ (-) and $\beta=180$ (- - -) are found to be slightly different. This is reproducible and reflects the fact that these spectra are first-order approximations to the actual tensor components for $\mathbf{E} \| b$. The neglected corrections are expected to be of the order of these differences.

This anisotropy can be compared to that obtained at single wavelengths in thin films with molecules oriented parallel to the substrate surface and also to the static dielectric constants, which we estimate for single crystal material by extrapolating our data to the long wavelength range with a fit to a Sellmeier dependence. These values are collected in Table 1.

Both the anisotropy and the absolute values at optical wavelengths are smaller for the films investigated in $[14,15]$, which can be taken indicative of less compact material in the films compared to the single crystal. The presence of voids would both reduce the values of $n$ and of the absolute anisotropy $\Delta n$. The effective in-plane optical functions published for similar films [16] are also much lower than our single-crystal data. However, we believe that this is not an intrinsic effect of film

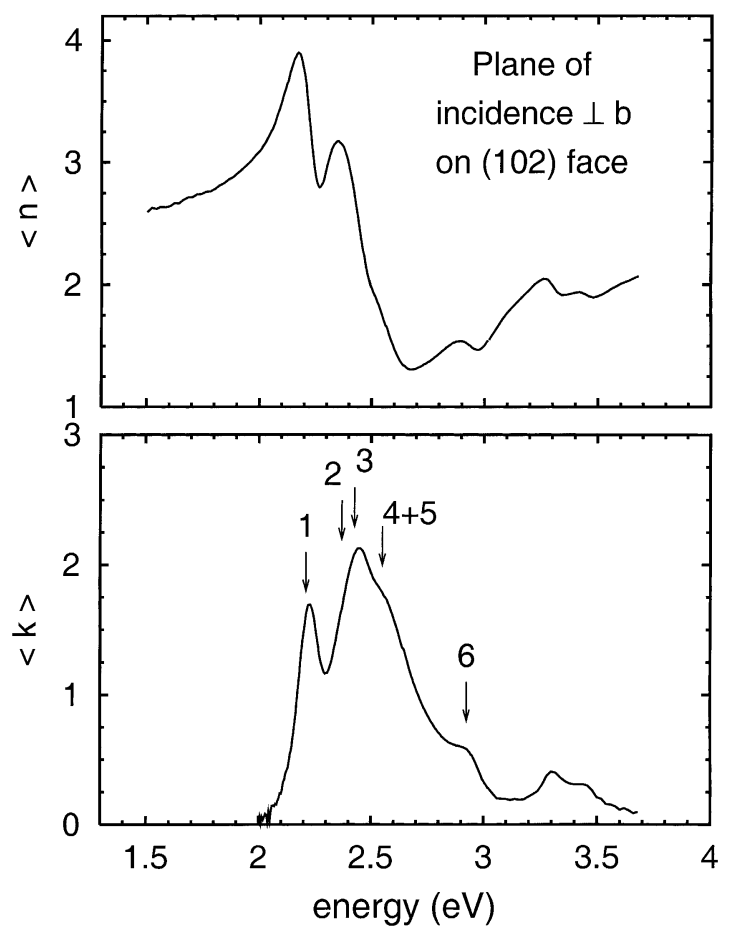

Fig. 5. Spectra from a (102) face with the plane of incidence perpendicular to the $b$-axis. In this orientation the measurements for sample azimuths $\beta=90$ and $\beta=270$ are found to coincide because of the (glide) mirror symmetry.

Table 1

Indices of refraction for PTCDA

\begin{tabular}{|c|c|c|c|c|c|c|c|}
\hline \multirow[b]{2}{*}{$\lambda(\mathrm{nm})$} & \multicolumn{3}{|c|}{ This work } & \multicolumn{2}{|c|}{ Ref. [14] } & \multicolumn{2}{|c|}{ Ref. [15] } \\
\hline & $\left\langle n_{\alpha}\right\rangle$ & $\left\langle n_{\beta}\right\rangle$ & $\left\langle n_{\gamma}\right\rangle$ & $n_{\perp}$ & $n_{\|}$ & $n_{\perp}$ & $n_{\|}$ \\
\hline 830 & 1.60 & 2.57 & 2.59 & & & 1.51 & 1.93 \\
\hline 1060 & 1.58 & 2.45 & 2.43 & 1.36 & 2.02 & & \\
\hline$\infty$ & 1.55 & 2.30 & 2.24 & $1.38^{\mathrm{a}}$ & $2.12^{\mathrm{a}}$ & & \\
\hline
\end{tabular}

The spectra of this work are extrapolated to long wavelengths $(\lambda \sim \infty)$ using a Sellmeier dispersion for $n$, thus giving a lower bound of the static dielectric constants.

${ }^{\mathrm{a}}$ From capacitance measurements between $100 \mathrm{~Hz}$ and 10 $\mathrm{MHz}$.

samples because we have measured effective optical functions in films that were closer to singlecrystal in-plane values. In fact, films are known to consist frequently of a mixture of $\alpha$ and $\beta$ phases $[6,17]$, with variable proportions depending upon preparation conditions. These results underline that for the values of optical functions to be 
meaningful, it is essential to simultaneously have a thorough knowledge of the structural parameters of the studied samples.

Whereas the spectra in the molecular planes show the familiar features similar to those known from PTCDA concentrated solutions and films $[2,18]$, the out-of-plane spectrum along the stacking direction (Fig. 3) is very different. The most prominent feature is a sharp peak close to $2.9 \mathrm{eV}$ that must be ascribed to a charge transfer transition. The two in-plane spectra (plane of incidence $\| b$ and $\perp b$ ) also show very important differences, indicative of formation of coherent excitons with clear Davydov splittings, that is, different transition energies depending on the polarization. In the following, we will concentrate on the properties in these molecular planes.

\section{Excitonic features}

The dielectric function of a solid reflects its electronic structure. In PTCDA the features that we observe in $\varepsilon(\omega)$ in the measured range should be caused by excitons. Excitonic transitions can be represented by a generalized Lorentzian line shape,

$\varepsilon(\omega)=C-A \mathrm{e}^{\mathrm{i} \theta}(\hbar \omega-E+\mathrm{i} \gamma)^{-1}$,

where $A$ is the amplitude, $\theta$ the phase angle, $E$ the transition energy, and $\gamma$ the broadening. These parameters can be determined by evaluating experimental $\langle\varepsilon(\omega)\rangle$ spectra using Eq. (1). Usually, fitting procedures are based on numerically calculated derivatives of $\langle\varepsilon(\omega)\rangle$. Here, we calculated the $\mathrm{d}^{2}\langle\varepsilon\rangle / \mathrm{d} \omega^{2}$ of our experimental spectra using the standard technique of smoothing polynomials. The appropriate polynomial degree and the number of correlated points were chosen such as to avoid line shape distortion, while giving the best possible structure enhancement [19].

The spectra of the (1 02 ) plane of PTCDA are particularly relevant because it coincides with the planes of the two symmetry-equivalent molecules to within $5^{\circ}$. Polarized optical spectra probe the transition dipole components in the measured plane. Our experiments showed that the most prominent spectral features are in-plane, whereas the peak close to $2.9 \mathrm{eV}$ (see Fig. 3) is an out-ofplane signature. For PTCDA, due to the crystal structure, the polarization directions of pure Frenkel (FE) states should essentially lie in the (102) lattice plane. In contrast, charge transfer (CT) states are expected to have substantial out-of plane polarization. (An eventual in-plane CT contribution would appear mixed with the FE states and, in principle, the observed transition dipoles in the (102) plane could correspond to such a mixture.)

In the derivative spectra, as illustrated in Fig. 6, we can deconvolute the different components of the main in-plane absorption band, where we observe six structures that are arbitrarily designated by numbers. The first exciton (labeled 1) is well separated from the other structures. A clear angular dependence of the energy and the amplitude fitted using Eq. (1) in different spectra, measured for $0 \leqslant \beta \leqslant 360^{\circ}$, is observed, as plotted in Fig. 7. The next four peaks reflect the complicated vibronic progression of the first peak with contributions of several mode combinations [20]. The sixth feature corresponds to the a-polarized peak at $2.9 \mathrm{eV}$ which, when fitted with Eq. (1) as function of $\beta$ displays a dependence of the phase angle $\theta$ on the sample azimuth which is absent in the other peaks. This variation $\theta(\beta)$ reflects an apparent line shape change caused by the out-of-plane polarization

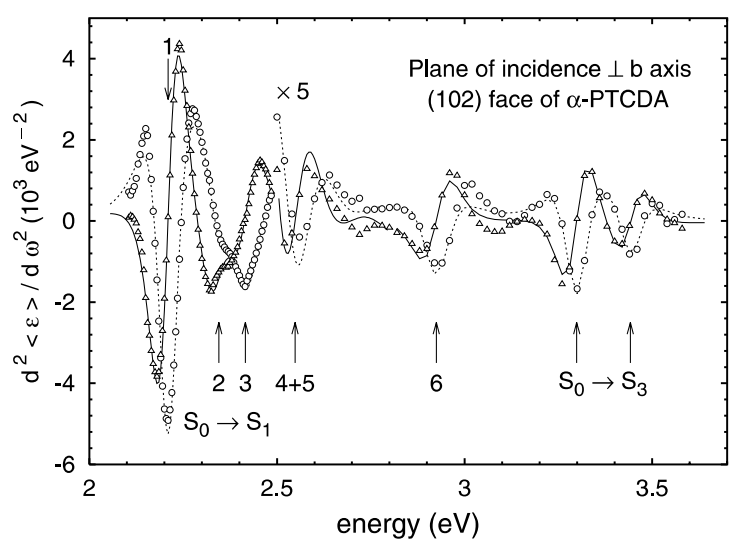

Fig. 6. Second-derivative spectrum of the polarization $\perp b$ axis. Experimental points are plotted by symbols $(\triangle$ : real part, $\bigcirc$ : imaginary part) and their best fits are given by lines. The arrows mark the obtained transition energies. 

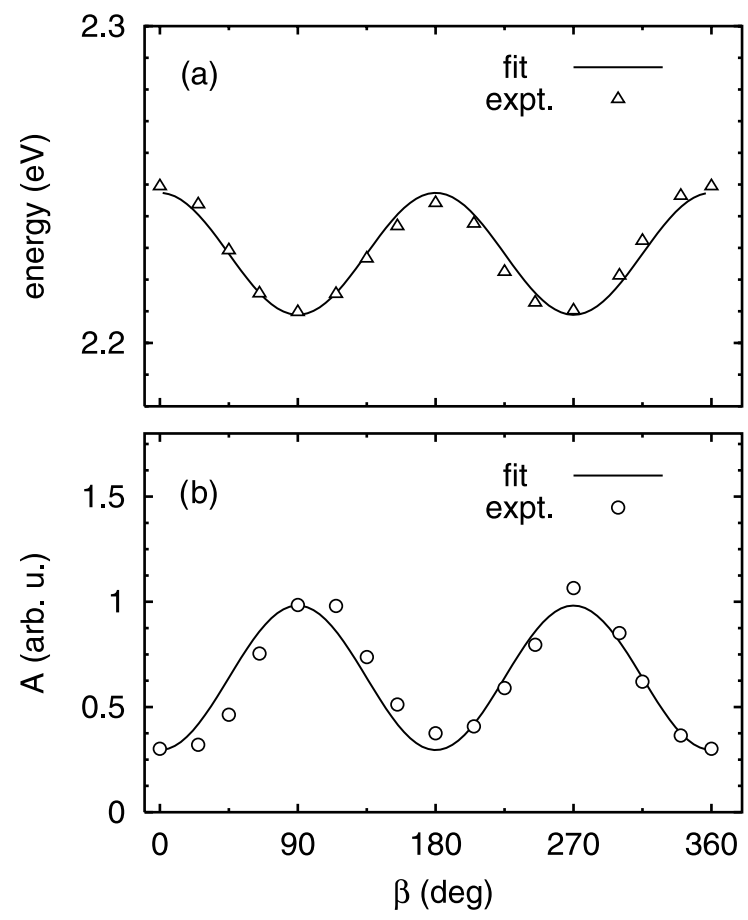

Fig. 7. Energy and amplitude of the first exciton observed as function of sample azimuth $\beta$. The origin $\beta=0$ means along the $b$-axis. Notice that the stronger Davydov component is that with lowest energy.

component. All other peaks are always well represented by $\theta=0$, consistent with their main in-plane character. Even in the second derivatives, peaks number 4 and 5 are not well resolved (not individually discernible). Therefore we chose to fit them together as a single structure. The obtained $\gamma$ has a clear dependence on $\beta$ : it oscillates from 110 to 200 $\mathrm{meV}$, indicating the presence of two close peaks with different azimuthal dependences. The polarization behaviour of peaks $2-5$ is rather complex and shall not be discussed here in detail. At higher energy there are two less intense, but clear transitions at 3.30 and $3.45 \mathrm{eV}$ that appear in all spectra. These are attributed to the $\mathrm{S}_{0} \rightarrow \mathrm{S}_{3}$ transition [20].

We now take a closer look at the anisotropic in-plane behaviour of the lowest-energy excitonic peak. For both its energy and its amplitude we observe an azimuthal dependence with a period of half a complete rotation $\left(180^{\circ}\right)$. Accordingly, the data of Fig. 7 can be represented by a dependence on $\beta$ of the kind
$f(\beta)=A(1+B \cos 2 \beta)$,

where $A$ and $B$ are parameters that reflect the mean value of the function $f$ and its modulation, respectively. Different theoretical scenarios have been used to represent the excitonic states of PTCDA. The character of the lowest-energy exciton is not yet well established. Different possibilities from Wannier-like, across CT to FE, and their combinations, have been considered [21-23]. A Wannier-CT combination giving a rather extended and nearly spherical exciton [23] cannot explain the observed anisotropy. On the contrary, CT-FE mixed states [21] as well as pure FE excitons [22] could produce anisotropic in-plane optical spectra. Both these approaches $[21,22]$ contain several adjustable parameters whose values must be obtained from fits to experimental data. Regarding anisotropy no experimental values were available, however, before our work [4].

In the approach of Hoffmann et al. [21] the anisotropy is attributed to the varying contribution of the CT excitonic character to the observed transition dipoles in the (llll 02 ) plane. In this view, the modulation parameter $B$ of Eq. (2) for the amplitude (see Fig. 7(b)) has a physical meaning. It can be expressed as $B=\cos \phi^{\prime}$, where $\phi^{\prime}$ represents the angle between the total transition dipoles of the two inequivalent molecular stacks. For a pure FE state, we should obtain $\phi^{\prime}=\phi=82.2^{\circ}$, which is the angle between the two molecules in the unit cell. However, for our measured lowest-energy exciton data we obtain $\phi^{\prime}=122.5 \pm 3.5^{\circ}$, that would indicate a large admixture of $\mathrm{CT}$ character. This result, derived using [21], is in striking contrast to the approach of Vragović et al. [22] since they obtain an anisotropy by considering only FE character. Both calculations give the polarization ratios or ratio of oscillator strength of differently polarized transitions. Our experimental results, presented in Fig. 7(b) and Table 2, agree qualitatively with the latter [22]: The oscillator strength of the transition labeled 1 is larger in the $\mathbf{E} \perp b$ than in the $\mathbf{E} \| b$ configuration. The opposite sign is found in the former approach [21]. However, the crucial difference between these two calculations is not merely the assumed microscopic nature of the excitonic states, but their symmetry and the fact 
Table 2

Experimental parameters obtained for the main absorption band of PTCDA from fits using Eq. (1)

\begin{tabular}{lllll}
\hline PTCDA band $\mathrm{S}_{0} \rightarrow \mathrm{S}_{1}$ & 1 & 2 & 3 & $4+5$ \\
\hline$E_{\mathrm{p}}(\mathrm{eV})$ & 2.247 & 2.37 & 2.490 & 2.617 \\
$E_{\mathrm{s}}(\mathrm{eV})$ & 2.210 & 2.37 & 2.427 & 2.550 \\
$A_{\mathrm{p}}\left(\mathrm{eV}^{-1}\right)$ & 0.34 & 0.16 & 0.56 & 3.40 \\
$A_{\mathrm{s}}\left(\mathrm{eV}^{-1}\right)$ & 1.03 & 0.64 & 0.75 & 0.69 \\
$\gamma_{\mathrm{p}}(\mathrm{meV})$ & 75 & 90 & 90 & 200 \\
$\gamma_{\mathrm{s}}(\mathrm{meV})$ & 75 & 90 & 90 & 110 \\
$\Delta E(\mathrm{meV})$ & 37 & - & 63 & 67 \\
\hline
\end{tabular}

The phase angle of these peaks is $\theta=0$. The subscripts $\mathrm{p}$ and $\mathrm{s}$ stand for transitions polarized parallel and perpendicular to the $b$-axis, respectively. $\Delta E=E_{\mathrm{p}}-E_{\mathrm{s}}$ is the Davydov splitting.

that Vragovic et al. treat the interactions between the two different molecules in the unit cell in the same framework as the coupling among stack neighbors, which in fact is stronger. In this approach [22] the full crystal geometry is approximately taken into account.

Regarding the sample azimuthal dependence of the energy plotted in Fig. 7(a), we observe a gradual variation without a change of the lineshape. We attribute the difference between the maximum and the minimum energy to Davydov splitting and evaluate $\Delta E=37 \mathrm{meV}$. The FWHM $(2 \gamma)$ of these excitonic lines is $150 \mathrm{meV}$. Since $\Delta E>2 \gamma$, if there were two different peaks it would not really be possible to resolve them separately. However, the fact that at all angles $\beta$ the observed peaks are symmetric and undistorted rather indicates a single component, that is, mixed modes for wave vectors of the light in intermediate $\beta$ positions, i.e., directional dispersion [24]. Knowledge of the full dielectric tensor, whose determination is currently underway [12], will finally allow to calculate light propagation in any arbitrary crystal direction.

\section{Summary}

We have presented an experimental study of the anisotropic optical properties of PTCDA. These have been measured by spectroscopic ellipsometry on single crystal samples at room temperature in the energy range from 1.5 to $3.7 \mathrm{eV}$. Relevant pseudo-dielectric functions have been obtained from complex reflectance ratios measured in highly symmetric configurations.

The dominant spectral features are shown to be mainly polarized in the molecular planes. A relatively narrow absorption peak at $\sim 2.9 \mathrm{eV}$ with a transition dipole moment approximately normal to the molecular planes has been attributed to a charge transfer transition. Its oscilator strength is ca 0.1 that of the in-plane exciton transitions close to $2.2 \mathrm{eV}$. The in-plane polarized transition dipole components obtained in the (102) crystallographic plane have been analyzed from numerically obtained $\mathrm{d}^{2}\langle\varepsilon\rangle / \mathrm{d} \omega^{2}$ spectra. Our results have been compared to the different electronic-structure calculations presently available $[21,22]$. From this comparison it follows that full understanding of the electronic structure of PTCDA not only requires to take into account the stronger interactions between stack neighbors but also those between the two symmetry-equivalent molecules in the unit cell. The formation of coherent excitonic states in the crystal with Davydov splittings is experimentally demonstrated. Further, the anisotropic behaviour of the lowest energy exciton is consistent with a dominating Frenkel character. This point, however, needs complementary experimental confirmation, as well as reconsideration (in view of the present experimental data) of the parameters used in the published theories.

A precise knowledge of the optical functions $\langle n\rangle$ and $\langle k\rangle$ of PTCDA is also an indispensable basis for further studies of the optical properties of films and of structures based on this compound.

\section{Acknowledgements}

We are grateful to Prof. H. Dosch for generous support and to $\mathrm{H}$. Port for critical reading of the manuscript.

\section{References}

[1] S.R. Forrest, Chem. Rev. 97 (1997) 1793.

[2] V. Bulović, P.E. Burrows, S.R. Forrest, J.A. Cronin, M.E. Thompson, Chem. Phys. 210 (1996) 1. 
[3] D.E. Aspnes, in: E.D. Palik (Ed.), Handbook of Optical Constants of Solids, Vol. 1, Academic Press, New York, 1985, pp. 89-112.

[4] M. Garriga, M.I. Alonso, N. Karl, J.O. Ossó, F. Schreiber, H. Dosch, Presented at DPG Frühjahrstagung, Hamburg 2001, see abstract in Verhandl. DPG (VI) 36 (1/2001), p. 506.

[5] N. Karl, in: R. Farchioni, G. Grosso (Eds.), Organic Electronic Materials, Springer, Berlin, 2001, p. 223.

[6] B. Krause, A.C. Dürr, K.A. Ritley, F. Schreiber, H. Dosch, D. Smilgies, Appl. Surf. Sci. 175-176 (2001) 332.

[7] A.J. Lovinger, S.R. Forrest, M.L. Kaplan, P.H. Schmidt, T. Venkatesan, J. Appl. Phys. 55 (1984) 476;

A.J. Lovinger, S.R. Forrest, M.L. Kaplan, P.H. Schmidt, T. Venkatesan, Bull. Am. Phys. Soc. 28 (1983) 364/476, a full crystal structure data set was kindly made available by M.L. Kaplan, C.S. Day, A.J. Lovinger, P.H. Schmidt, S.R. Forrest.

[8] M. Möbus, N. Karl, T. Kobayashi, J. Cryst. Growth 116 (1992) 495.

[9] T. Ogawa, K. Kuwamoto, S. Isoda, T. Kobayashi, N. Karl, Acta Cryst. B 55 (1999) 123.

[10] F.F. So, S.R. Forrest, Y.Q. Shi, W.H. Steier, Appl. Phys. Lett. 56 (1990) 674.

[11] M.I. Alonso, M. Garriga, F. Alsina, S. Piñol, Appl. Phys. Lett. 67 (1995) 596.

[12] M. Garriga et al., unpublished.
[13] D.E. Aspnes, J. Opt. Soc. Am. 70 (1980) 1275.

[14] D.Y. Zang, F.F. So, S.R. Forrest, Appl. Phys. Lett. 59 (1991) 823.

[15] H. Fuchigami, S. Tanimura, Y. Uehara, T. Kurata, S. Tsunoda, Jpn. J. Appl. Phys. 34 (1995) 3852.

[16] A.B. Djurišić, T. Fritz, K. Leo, Opt. Commun. 183 (2000) 123.

[17] M. Leonhardt, O. Mager, H. Port, Chem. Phys. Lett. 313 (1999) 24.

[18] U. Gómez, M. Leonhardt, H. Port, H.C. Wolf, Chem. Phys. Lett 268 (1997) 1.

[19] P. Lautenschlager, M. Garriga, S. Logothetidis, M. Cardona, Phys. Rev. B 35 (1987) 9174.

[20] K. Gustav, M. Leonhardt, H. Port, Monatsh. Chem. 128 (1997) 105.

[21] M. Hoffmann, K. Schmidt, T. Fritz, T. Hasche, V.M. Agranovich, K. Leo, Chem. Phys. 258 (2000) 73; M. Hoffmann, PhD Thesis, Technische Universität Dresden, 2000.

[22] I. Vragović, R. Scholz, M. Schreiber, Europhys. Lett. 57 (2002) 288. (preprint, kindly made available by the authors).

[23] Z. Shen, P.E. Burrows, S.R. Forrest, M. Ziari, W.H. Steier, Chem. Phys. Lett. 236 (1995) 129.

[24] G. Weiser, W. Fuhs, H.J. Hesse, Chem. Phys. 52 (1980) 183. 